\title{
On Propagation Characteristics of Resilient Functions
}

\author{
Pascale Charpin ${ }^{1}$ and Enes Pasalic ${ }^{2}$ \\ 1 INRIA, projet CODES, Domaine de Voluceau, Rocquencourt, BP 105, \\ 78153 Le Chesnay Cedex, France. \\ Pascale.Charpin@inria.fr \\ 2 Department of Information Technology, Lund University \\ P.O. Box 118, 22100 Lund, Sweden. \\ enes@it.1th.se
}

\begin{abstract}
In this paper we derive several important results towards a better understanding of propagation characteristics of resilient Boolean functions. We first introduce a new upper bound on nonlinearity of a given resilient function depending on the propagation criterion. We later show that a large class of resilient functions admit a linear structure; more generally, we exhibit some divisibility properties concerning the Walsh-spectrum of the derivatives of any resilient function. We prove that, fixing the order of resiliency and the degree of propagation criterion, a high algebraic degree is a necessary condition for construction of functions with good autocorrelation properties. We conclude by a study of the main constructions of resilient functions. We notably show how to avoid linear structures when a linear concatenation is used and when the recursive construction introduced in [11] is chosen.
\end{abstract}

Keywords: Boolean functions, nonlinearity, propagation characteristics, resiliency, linear space.

\section{Introduction}

The security of most conventional cryptographic systems is based on some properties of Boolean functions - currently called cryptographic criteria. This paper deals with well-known such criteria. The nonlinearity, the distance from a Boolean function to the set of all affine functions, prevents linear attacks in block ciphers [9]. Correlation-immune functions were first introduced by Siegenthaler [14] in order to construct running-key generators for stream ciphers which resist to correlation attack. A balanced such function is said to be resilient; resiliency appears as the main criterion in several systems (see, for instance, 1]). The propagation criterion (PC) was introduced by Preneel [12], generalizing the strict avalanche criterion [17]. More generally, the propagation characteristics of

\footnotetext{
${ }^{1}$ More detailed explanations, examples and full proofs can be found in the extended version of this paper, see [6].
} 
any Boolean function refer to certain properties of its derivatives [19]. A function which has constant derivatives is said to have a nontrivial linear space, the space of its linear structures. The distance from a Boolean function to the set of functions with linear structures was explained by Meier and Staffelbach in [10].

Recently, the relationships between propagation characteristics, nonlinearity, and correlation-immunity were investigated (see notably [2, [13, [15, 20], 4]). Generally, in all recent works, it appears that good cryptographic properties imply that the given function belongs to some well-structured class. It is especially true for resilient functions; a few effective constructions are known and the main of these are based on concatenations 7,16 . Our main purpose is the study of the consequences of high resiliency for other cryptographic criteria. How high resiliency could lead to some weakness? In accordance with [10], such weakness has to be considered up to any simple transformation (for instance, any affine transformation).

In Section 2, we present the main tools for the study of Boolean functions on $\mathbb{F}_{2}^{n}$, the basic definitions and some recent results concerning the cryptographic criteria. In Section 3 we consider resilient functions which satisfy a certain propagation criterion. We introduce a new nontrivial upper bound on the nonlinearity of $t$-resilient functions satisfying PC with respect to some subspace of dimension $p$ (Theorem 3). We then emphasize that for a fixed order of resiliency, the upper bound on nonlinearity of $f$, is smaller for larger $p$. Section 4 is devoted to the characterization of the linear space of functions. Different criteria regarding the functions with linear structures are addressed here. We then deduce that high resiliency leads to the existence of linear structures (Corollary 2). In Section 5] we study the weights of the derivatives of resilient functions which satisfy (or not) some propagation criterion. Our results reinforce those of the previous section. Namely, high resiliency leads to high divisibility for the weights of derivatives; moreover, taking into account the degree of propagation and the degree of the function, this divisibility increases (Theorem [5). In Section 6 we discuss the main known constructions of resilient functions. We first characterize resilient functions, obtained by linear concatenation, which have no linear structure (Proposition [3). We later study two recursive constructions [16], [1]. We prove that the first one provides resilient functions which have a linear space not reduced to 0 , while the second one preserves the lack of linear structure (Proposition 7).

\section{Definitions and Basic Properties}

\subsection{Boolean Functions}

We denote by $\mathcal{B}_{n}$ the set of Boolean functions of $n$ variables. Thus $f \in \mathcal{B}_{n}$ is a function from $\mathbb{F}_{2}^{n}$ to $\mathbb{F}_{2}$; it is generally represented by its algebraic normal form:

$$
f\left(x_{1}, \ldots, x_{n}\right)=\sum_{u \in \mathbb{F}_{2}^{n}} \lambda_{u}\left(\prod_{i=1}^{n} x_{i}^{u_{i}}\right), \quad \lambda_{u} \in \mathbb{F}_{2} .
$$


The degree of $f$ is the maximal value of the Hamming weight of $u$ such that $\lambda_{u} \neq 0$. The linear functions will be represented by means of the scalar product, with respect to the standard basis. They will be denoted as follows: for any $\alpha \in \mathbb{F}_{2}^{n}, \varphi_{\alpha}: x \in \mathbb{F}_{2}^{n} \longmapsto \alpha \cdot x=\sum_{i=1}^{n} \alpha_{i} x_{i}$.

The Walsh transform of $f \in \mathcal{B}_{n}$ in point $\alpha$ is denoted $\mathcal{F}\left(f+\varphi_{\alpha}\right)$ and calculated as,

$$
\alpha \in \mathbb{F}_{2}^{n} \longmapsto \mathcal{F}\left(f+\varphi_{\alpha}\right)=\sum_{x \in \mathbb{F}_{2}^{n}}(-1)^{f(x)+\varphi_{\alpha}(x)} .
$$

The values of these coefficients form the Walsh-spectrum of $f$. For convenience, $\mathcal{F}(f)$ will denote the Walsh transform in $\alpha=0$. The nonlinearity $\mathcal{N}_{f}$ of $f \in \mathcal{B}_{n}$ is related to the Walsh transform via following expression:

$$
\mathcal{N}_{f}=2^{n-1}-\frac{\mathcal{L}(f)}{2} \quad \text { where } \quad \mathcal{L}(f)=\max _{\alpha \in \mathbb{F}_{2}^{n}}\left|\mathcal{F}\left(f+\varphi_{\alpha}\right)\right|
$$

The propagation characteristics of $f$ are described by the behavior of its derivatives. The derivative of $f \in \mathcal{B}_{n}$ with respect to any direction $a \in \mathbb{F}_{2}^{n}$, is the mapping $D_{a} f: x \longmapsto f(x)+f(a+x)$. Thus, the auto-correlation function of $f$ refers to the function $a \mapsto \mathcal{F}\left(D_{a} f\right)$. The main indicators of propagation characteristics are the absolute indicator and the sum-of-square indicator [19]:

$$
\mathcal{M}(f)=\max _{a \in \mathbf{F}_{2}^{m}, a \neq 0}\left|\mathcal{F}\left(D_{a} f\right)\right| \text { and } \mathcal{V}(f)=\sum_{a \in \mathbf{F}_{2}^{m}} \mathcal{F}^{2}\left(D_{a} f\right) .
$$

For any linear subspace $V$ of $\mathbb{F}_{2}^{n}$, its dual $V^{\perp}$ will be the subspace of elements $x \in \mathbb{F}_{2}^{n}$ such that $x \cdot y=0$ for all $y \in V$. The next formula provides a link between the Walsh and autocorrelation spectra of $f$. The proof can be found in [3. Lemma V.2].

Lemma 1 Let $V$ be a linear subspace of $\mathbb{F}_{2}^{n}$ of dimension $k$. Then for any function $f$ in $\mathcal{B}_{n}$ we have for any $\beta \in \mathbb{F}_{2}^{n}$,

$$
\sum_{v \in V} \mathcal{F}^{2}\left(f+\varphi_{\beta+v}\right)=2^{k} \sum_{u \in V^{\perp}}(-1)^{\beta \cdot u} \mathcal{F}\left(D_{u} f\right) .
$$

For $V=\{0\}$, (11) becomes the well-known relation:

$$
\mathcal{F}^{2}\left(f+\varphi_{\beta}\right)=\sum_{u \in \mathbb{F}_{2}^{n}}(-1)^{\beta \cdot u} \mathcal{F}\left(D_{u} f\right)
$$

\subsection{Resiliency and Propagation Characteristics}

The next definitions are now classical. They were introduced in [10,12] (for the propagation characteristics) and in [1418] (for the resiliency). Recall that the Hamming weight of any binary vector $y$ is $w t(y)=\#\left\{i \mid y_{i}=1\right\}$, where $\# A$ denotes the cardinality of any set $A$. By convention, the weight of $f \in \mathcal{B}_{n}$ is the Hamming weight of its corresponding codeword, where the codeword of $f(x)$ 
is the sequence of values $f(x)$, when $x$ runs through $\mathbb{F}_{2}^{n}$. Any function $f \in \mathcal{B}_{n}$ is balanced when $w t(f)=2^{n-1}$ or, equivalently, $\mathcal{F}(f)=0$. A function exhibits good propagation characteristics when its autocorrelation function takes "small" (absolute) values; therefore the related indicators have to be "small" [19].

Definition 1 The linear space of any Boolean function $f$ is the linear subspace of those elements a such that the function $D_{a} f$ is constant. Such nonzero a is called a linear structure of $f$.

The function $f \in \mathcal{B}_{n}$ has a linear structure if and only if $\mathcal{M}(f)$ takes its maximal value $2^{n}$. On the other hand the sum-of-square indicator provides a bound for the nonlinearity.

Theorem 1 [2] Let $f \in \mathcal{B}_{n}$. Then we have $\mathcal{V}(f) \leq 2^{n} \mathcal{L}^{2}(f)$ with equality if and only if the Walsh spectrum of $f$ takes at most three values, $0, \mathcal{L}(f)$ and $-\mathcal{L}(f)$.

The propagation criterion of $f$ concerns the set of balanced derivatives.

Definition 2 Let $E \subset \mathbb{F}_{2}^{n}$. The function $f \in \mathcal{B}_{n}$ satisfies the propagation criterion (PC) with respect to $E$ if for all $e \in E$ the function $D_{e} f$ is balanced. The function $f$ satisfies $\mathrm{PC}$ of degree $p(\mathrm{PC}(p))$ for some positive integer $p$ when $D_{a} f$ is balanced for any $a \in \mathbb{F}_{2}^{n}$ such that $1 \leq w t(a) \leq p$.

The correlation-immunity is characterized by the set of zero values in the Walsh spectrum.

Definition 3 Let $f \in \mathcal{B}_{n}$ and let $t$ be some positive integer. The function $f$ is said to be correlation-immune of order $t$ if and only if $\mathcal{F}\left(f+\varphi_{\alpha}\right)=0$ for any $a \in \mathbb{F}_{2}^{n}$ such that $1 \leq w t(\alpha) \leq t$. Moreover, when $f$ is balanced, it is said to be t-resilient. A balanced function is said to be 0-resilient.

Besides its maximum value, the whole Walsh spectrum of a Boolean function has a great cryptographic significance. Several recent works are devoted to the divisibility of the Walsh coefficients of resilient functions. Sarkar and Maitra proved in [13] that any $t$-resilient function $f \in \mathcal{B}_{n}$ satisfies for all $\alpha: \mathcal{F}\left(f+\varphi_{\alpha}\right) \equiv 0$ $\left(\bmod 2^{t+2}\right)$. This result has been independently obtained by Tarannikov [15]. It was improved by Carlet in [4] by including the algebraic degree $d$ of the function:

$$
\mathcal{F}\left(f+\varphi_{\alpha}\right) \equiv 0 \quad\left(\bmod 2^{t+2+\left\lfloor\frac{n-t-2}{d}\right\rfloor}\right), \forall \alpha \in \mathbb{F}_{2}^{n}
$$

(where $\lfloor r\rfloor$ denotes the integer part of $r$ ). Carlet then derived a new upper bound on $\mathcal{N}_{f}$,

$$
\mathcal{N}_{f} \leq 2^{n-1}-2^{t+1+\left\lfloor\frac{n-t-2}{d}\right\rfloor} .
$$

This bound is lose for small $t$, since there is a tighter upper bound derived from the nonlinearity of bent functions. However, for $t>\frac{n}{2}-2$ the bound above is tighter for any $n$. 


\section{On Resilient Boolean Functions Satisfying PC}

In this section, we focus on an improvement of the bound (3) when considering any $t$-resilient function which moreover satisfies some propagation criterion.

\subsection{Preliminary}

In a recent paper, Zhang and Zheng introduced several properties regarding the relationship between the correlation-immunity and propagation criteria 20]. They begin by giving a lower bound for the nonlinearity of functions satisfying $\mathrm{PC}(p)$. The following result is given in 20 , Theorem 1].

Theorem 2 Let $f$ be a non-bent function in $\mathcal{B}_{n}$ satisfying $\operatorname{PC}(p)$. Then the nonlinearity of $f$ satisfies

$$
\mathcal{N}_{f} \geq 2^{n-1}-2^{n-\frac{p}{2}-1} \quad \text { or, equivalently, } \quad \mathcal{L}(f) \leq 2^{n-\frac{p}{2}} .
$$

Moreover, if $\mathcal{L}(f)=2^{n-\frac{p}{2}}$, then $p=n-1$ and $n$ is odd.

Actually this lower bound can be established more generally.

Proposition 1 Let $f$ be a non-bent function in $\mathcal{B}_{n}$. Assume that $f$ satisfies $P C$ with respect to $U \backslash\{0\}$, where $U$ is a subspace of $\mathbb{F}_{2}^{n}$ of dimension $p$. Then the nonlinearity of $f$ satisfies (4). This especially holds when $f$ satisfies $P C(p)$. When $p=n-1$, for odd $n$, or $p=n-2$, for even $n$, then $\mathcal{L}(f)=2^{n-\frac{p}{2}}$.

Proof: We apply (1) with $k=n-p$ and $V=U^{\perp}$. Then for any $\beta$,

$$
\sum_{v \in U^{\perp}} \mathcal{F}^{2}\left(f+\varphi_{\beta+v}\right)=2^{n-p} \mathcal{F}\left(D_{0} f\right)=2^{2 n-p} .
$$

This implies that $\mathcal{L}^{2}(f) \leq 2^{2 n-p}$ or, equivalently, that $\mathcal{N}_{f} \geq 2^{n-1}-2^{\frac{2 n-p}{2}-1}$. When the function $f$ satisfies $\mathrm{PC}(p)$, it satisfies $\mathrm{PC}$ with respect to $U_{a} \backslash\{0\}$ for any $a$ where

$$
U_{a}=\left\{u \in \mathbb{F}_{2}^{n} \mid \preccurlyeq a\right\} \text { with } w t(a)=p .
$$

Note that $u \preccurlyeq a$ means that $a$ covers $u$, i.e., $u_{i} \leq a_{i}$ for all $i$ in the range $[1, n]$.

The cases $p=n-1$ and $p=n-2$ were explained in [3. $\S$ V.C]. Note that, according to the previous theorem, it is impossible to have: $f$ satisfies $\mathrm{PC}(n-2)$ and $\mathcal{L}(f)=2^{n-\frac{p}{2}}$.

With the hypothesis of Theorem 2, $\mathcal{L}(f)=2^{n-\frac{p}{2}}$ implies that $p=n-1$ for odd $n$ (and that $f$ is bent for even $n$ ). The functions satisfying $\operatorname{PC}(n-1)$ were fully-characterized in [2]. Such a function $f$ admits one and only one linear structure, say $e$, and is such that $D_{a} f$ is balanced unless $a \in\{0, e\}$. Moreover it cannot be 1-resilient, with respect to any basis. In the case of Proposition 1 . it is possible to have $\mathcal{L}(f)=2^{n-\frac{p}{2}}$ for any even $p$. Furthermore for $p=n-1$, when $f$ is balanced it is generally 1-resilient, with respect to some basis (see [2], Corollary 2, Theorems 4 and 7 ). 
In the sequel of this section, we will consider functions which are $t$-resilient with respect to the standard basis. We will fix the basis for the definitions of the $t$-resiliency; for the PC property we consider particular subspaces which are defined by means of this standard basis. We first indicate some restriction on the sum $p+t$.

Lemma 2 Let $f$ be a t-resilient function satisfying $P C$ with respect to the nonzero elements of $U_{a}, w t(a)=p$, defined as in (5). Denote by $\bar{a}$ the vector $(1+$ $\left.a_{1}, \ldots, 1+a_{n}\right)$.

Then $p+t \leq n-1$. If $p+t=n-1$ and $\mathcal{F}\left(f+\varphi_{\bar{a}}\right) \neq 0$ then $\mathcal{L}^{2}(f)=$ $\mathcal{F}^{2}\left(f+\varphi_{\bar{a}}\right)=2^{2 n-p}$. When $p+t=n-1$ and $f$ satisfies $P C(p)$ then $p=n-1$, $n$ is odd and $t=0$.

Proof: We apply (11) with $k=n-p, V=U_{a}^{\perp}$ and $\beta=0: \sum_{v \preccurlyeq \bar{a}} \mathcal{F}^{2}\left(f+\varphi_{v}\right)=$ $2^{2 n-p}$.

Since $w t(\bar{a})=n-p$ and $f$ is $t$-resilient, $t \geq n-p$ would imply that each term in the sum above is zero, a contradiction. Now, assuming that $t=n-p-1$, there is only one possible non-zero term in this sum (for $v=\bar{a}$ ). According to Proposition $1 \mathcal{F}^{2}\left(f+\varphi_{\bar{a}}\right)=2^{2 n-p}$ provides $\mathcal{L}^{2}(f)=2^{2 n-p}$. The proof is completed by means of Theorem 2 ,

\subsection{A New Upper Bound}

We will show that there exists a nontrivial upper bound on the nonlinearity of $t$-resilient functions satisfying $\mathrm{PC}$ with respect to the nonzero elements of some subspace of dimension $p$. According to the previous discussion we will assume that $p+t=n-k$ with $k \geq 2$. Recall that the degree $d$ of any $t$-resilient function in $\mathcal{B}_{n}$ satisfies $d \leq n-t-1[14$.

Theorem 3 Let $f \in \mathcal{B}_{n}$ be at-resilient function of degree $d$ with $d \geq 2$. Assume that $f$ satisfies $P C$ with respect to $U_{a} \backslash\{0\}$ where $U_{a}=\left\{u \in \mathbb{F}_{2}^{n} \mid u \preccurlyeq a\right\}$ with $w t(a)=p$. Let $p+t=n-k$ with $2 \leq k \leq n-2$. Then the upper bound on nonlinearity of $f$ is given by,

$$
\mathcal{N}_{f} \leq 2^{n-1}-\ell 2^{t+1+\left\lfloor\frac{n-t-2}{d}\right\rfloor}
$$

where $\ell$ is the minimum integer among all positive integers $i$ satisfying

$$
i^{2} \sum_{j=1}^{k}\left(\begin{array}{l}
t+k \\
t+j
\end{array}\right) \geq 2^{p+2 k-4-2\left\lfloor\frac{n-t-2}{d}\right\rfloor} .
$$

This is especially true when $f$ satisfies $P C(p)$.

Proof: From Lemma 1 since $\mathcal{F}\left(D_{u} f\right)=0$ for any nonzero $u \in U_{a}$, we have:

$$
\sum_{v \in U_{a}^{\perp}} \mathcal{F}^{2}\left(f+\varphi_{v}\right)=2^{n-p} \mathcal{F}\left(D_{0} f\right)=2^{2 n-p},
$$


On the other hand we know that, as any $t$-resilient function, $f$ satisfies for all $\alpha: \mathcal{F}\left(f+\varphi_{\alpha}\right) \equiv 0\left(\bmod 2^{t+2+\epsilon}\right)$, where $\epsilon=\left\lfloor\frac{n-t-2}{d}\right\rfloor$ (see $\left.(2)\right)$. Combining this result with (8), we conclude that for any $v \in U_{a}^{\perp}$ there is an integer $i$ such that $0 \leq i^{2} \leq 2^{p+2 k-4-2 \epsilon}$ and $\mathcal{F}^{2}\left(f+\varphi_{v}\right)=i^{2} 2^{2(t+2+\epsilon)}$.

Remark that $2 n-p-2(t+2+\epsilon)=p+2 k-4-2 \epsilon$, since $p+t=n-k$, providing the upper bound on $i^{2}$. Moreover, the equality (8) implies $p+2 k-4-2 \epsilon \geq 0$. Now we set for any $i$ :

$$
\lambda_{i}=\operatorname{card}\left\{v \in U_{a}^{\perp}:\left|\mathcal{F}\left(f+\varphi_{v}\right)\right|=i 2^{t+2+\epsilon}\right\} .
$$

Then we may rewrite (8) in terms of $\lambda_{i}$. We obtain (where $c=2^{p+2 k-4-2 \epsilon}$ ):

$$
\sum_{i=1}^{c} \lambda_{i} i^{2} 2^{2 t+4+2 \epsilon}=2^{2 n-p}, \text { i.e., } \sum_{i=1}^{c} \lambda_{i} i^{2}=2^{p+2 k-4-2 \epsilon},
$$

On the other hand, we consider the number $\Lambda$ of nonzero coefficients $\mathcal{F}\left(f+\varphi_{v}\right)$ when $v$ describes $U_{a}^{\perp}$. Since $f$ is $t$-resilient, then $\Lambda \leq \sum_{j=1}^{k}\left(\begin{array}{c}t+k \\ t+j\end{array}\right)$. Thus we claim that from a certain positive value of $i$, say for all $i \geq i_{0}$, we have :

$$
\sum_{j=1}^{c} \lambda_{j} j^{2} \leq \Lambda i^{2} \leq i^{2} \sum_{j=1}^{k}\left(\begin{array}{l}
t+k \\
t+j
\end{array}\right)
$$

Therefore, we can define $\ell$ as the smallest integer such that $2^{p+2 k-4-2 \epsilon} \leq$ $\ell^{2} \sum_{j=1}^{k}\left(\begin{array}{c}t+k \\ t+j\end{array}\right)$. Moreover we are sure that there is some $v \in U_{a}^{\perp}$ such that $\left|\mathcal{F}\left(f+\varphi_{v}\right)\right| \geq \ell 2^{t+2+\epsilon}$, because if this is not true then we can define $i<\ell$ such that $\left|\mathcal{F}\left(f+\varphi_{v}\right)\right| \leq i 2^{t+2+\epsilon}$ for all $v$; such $i$ satisfies (9) contradicting the assumption. Thus we have proved that the maximal absolute value of the coefficients $\mathcal{F}\left(f+\varphi_{v}\right)$ is at least $\ell 2^{t+2+\epsilon}$ or, equivalently, that $\mathcal{N}_{f}$ satisfies (6).

According to the previous theorem, it is easy to see that for a fixed order of resiliency the upper bound on nonlinearity becomes smaller as $p$ increases. Note that the lower bound (4), which has concern with propagation criterion only, increases with $p$. The next example clearly indicate the trade-off between the nonlinearity and propagation. Another illustration is the following corollary, directly deduced from Theorem 3 (for $k=2$ ).

Corollary 1 Let $f$ be a Boolean function in $\mathcal{B}_{n}$ of degree d satisfying the hypothesis of Theorem [3. Furthermore, let $p+t=n-2, p>0$. Then the upper bound on nonlinearity is given by, $\mathcal{N}_{f} \leq 2^{n-1}-\ell 2^{t+1+\left\lfloor\frac{p}{d}\right\rfloor}$, where $\ell$ is the minimum integer among all positive integers $i$ satisfying

$$
i^{2}(t+3) \geq 2^{p-2\left\lfloor\frac{p}{d}\right\rfloor} .
$$

Example 1 Let $f$ be a 4 -resilient function in $\mathcal{B}_{10}$. Assume there is $a \in \mathbb{F}_{2}^{10}$ of weight $w t(a)=3$ such that $D_{u} f$ is balanced for any nonzero $u \preccurlyeq a$, i.e., $t=4$ 
and $p=3$ in Theorem 3 . We suppose that the degree $d$ of $f$ is such that $\epsilon=0$; for instance $d=5$.

We have $p+t=7$ and $k=3$. Thus $\ell$ is the smallest integer $i$ satisfying $i^{2} \sum_{j=1}^{3}\left(\begin{array}{c}7 \\ 4+j\end{array}\right) \geq 32$; so $\ell=2$. Then the nonlinearity of $f$ is less than or equal to $2^{n-1}-2 \cdot 2^{t+1}=448$, for $n=10, t=4, p=3$. We conclude that $\mathcal{N}_{f} \leq 448$, while the upper bound (3) gives $\mathcal{N}_{f} \leq 480$. Such a function, with these parameters, was firstly constructed in [11].

\section{On Functions with(out) Linear Structure}

To construct effectively functions with high resiliency remains an important open problem. However, high resiliency could imply some property which leads to some cryptographic weakness. This section is devoted to the existence of linear structures. We propose some general tools characterizing linear structures; then we can show that high resiliency provides linear structures. Recall that an attack on block ciphers, based on the existence of linear structures, was proposed by Evertse [8].

\subsection{On Distance to Linear Structures}

In [10, the propagation criterion was defined as the nonlinearity of $f$ with respect to a linear structure. Since this criterion is invariant under the general affine group, it was considered as a useful criterion. It allows us to quantify the distance of $f$ to any linear structure as we explain briefly.

Definition 4 Let $L S(n)$ denote the subset of Boolean functions having linear structures:

$$
L S(n)=\left\{g \in \mathcal{B}_{n} \mid \exists \text { a such that } D_{a} g \in\{0,1\}\right\} .
$$

The nonlinearity of $f$ with respect to the functions with linear structures is defined as,

$$
\sigma(f)=\min _{g \in L S(n)} w t(f+g) .
$$

Note that $L S(n)$ properly contains the set of all affine functions. Moreover, it contains quadratic functions which are not bent. Thus, this kind of nonlinearity is much stronger than the usual nonlinearity. In 10, it was also proved that $\sigma(f) \leq 2^{n-2}$ for $f \in \mathcal{B}_{n}$ with equality if and only if $f$ is bent. More precisely, the minimum distance of $f$ to the set of the functions which have a linear structure $a$ is less than or equal to $2^{n-2}$ with equality if and only if $D_{a} f$ is balanced.

\subsection{Criteria for Linear Structure}

A priori, there is no criteria to decide upon whether a Boolean function has a linear structure except of checking for all possible linear structures. However, as we will show, this problem for any function $f$, is strongly related with some properties of its Walsh-spectrum. 
Lemma 3 Let $f$ be a Boolean function in $\mathcal{B}_{n}$. Then $f$ has a linear structure, say $a$, if and only if either the hyperplane $\{0, a\}^{\perp}$ (if $D_{a} f=1$ ) or its complement (if $D_{a} f=0$ ) is contained in the set

$$
Z_{f}=\left\{\alpha \mid \mathcal{F}\left(f+\varphi_{\alpha}\right)=0\right\} .
$$

In particular, if the cardinality of $Z_{f}$ does not exceed $2^{n-1}-1$ then $f$ has no linear structure.

Proof: For any $a \neq 0$ we consider the hyperplane $H=\{0, a\}^{\perp}$; then we can write (1) as follows:

$$
\sum_{u \in H} \mathcal{F}^{2}\left(f+\varphi_{u}\right)=2^{n-1}\left(\mathcal{F}\left(D_{0} f\right)+\mathcal{F}\left(D_{a} f\right)\right)=2^{2 n-1}+2^{n-1} \mathcal{F}\left(D_{a} f\right) .
$$

Note that $a$ is a linear structure of $f$ if and only if either $\mathcal{F}\left(D_{a} f\right)$ ) $=2^{n}$ (when $D_{a} f=0$ ) or $\left.\mathcal{F}\left(D_{a} f\right)\right)=-2^{n}$ (when $D_{a} f=1$ ). We deduce from (12) that $D_{a} f=1$ if and only if $\mathcal{F}\left(f+\varphi_{u}\right)=0$ for all $u$ in $H$; on the other hand, $D_{a} f=0$ if and only if $\sum_{u \in H} \mathcal{F}^{2}\left(f+\varphi_{u}\right)=2^{2 n}$. But this last property means $\sum_{u \in \mathbb{F}_{2}^{n} \backslash H} \mathcal{F}^{2}\left(f+\varphi_{u}\right)=0$, because of Parseval's relation. So we have proved that $D_{a} f$ is constant if and only if the set $Z_{f}$ contains either $H$ or $\mathbb{F}_{2}^{n} \backslash H$. Therefore, the cardinality of $Z_{f}$ must be at least $2^{n-1}$ when $f$ has a linear structure, completing the proof.

Note that any $t$-resilient function $f \in \mathcal{B}_{n}$, with $t \geq\left\lfloor\frac{n}{2}\right\rfloor$, is such that the number of zero values in its Walsh spectrum is greater than or equal to $2^{n-1}$. Thus, for such a function, we cannot apply the previous lemma. An important consequence is that the design rule for $t<\frac{n}{2}$ may be formulated as: Construct a Boolean function $f \in \mathcal{B}_{n}$ by selecting an optimum choice of the design parameters of concern (nonlinearity, order of resiliency, PC degree) such that its Walsh spectrum contains less than $2^{n-1}$ zeros. Now the previous lemma yields a more practical condition.

Theorem 4 Let $f \in \mathcal{B}_{n}$. Then $D_{a} f \neq 0$ for any nonzero a if and only if $f$ satisfies $S(f)$ : there exists a basis $\left(e_{1}, \ldots, e_{n}\right)$ of $\mathbb{F}_{2}^{n}$ such that $\mathcal{F}\left(f+\varphi_{e_{i}}\right) \neq$ $0,1 \leq i \leq n$. Moreover

(i) when $f$ is not balanced, $f$ has no linear structure if and only if the condition $S(f)$ is satisfied;

(ii) when $f$ is balanced, $f$ has no linear structure if and only if there is $e \neq 0$ in $\mathbb{F}_{2}^{n}$ such that the function $g=f+\varphi_{e}$ is not balanced and the condition $S(g)$ is satisfied.

Proof: We assume that $f$ is neither affine nor constant. In accordance with Lemma $3 . D_{a} f=0$ for some nonzero $a$ if and only if $Z_{f}$ contains the complement of the hyperplane $\{0, a\}^{\perp}$. Let

$$
N Z_{f}=\left\{\alpha \mid \mathcal{F}\left(f+\varphi_{\alpha}\right) \neq 0\right\}
$$


be the complement of $Z_{f}$ in $\mathbb{F}_{2}^{n}$. Denote by $\bar{H}$ the complement of some hyperplane $H$. Clearly, $Z_{f}$ contains $\bar{H}$ if and only if $N Z_{f}$ is contained in $H$. More generally, $Z_{f}$ contains the complement of some hyperplane if and only if the rank of the set $N Z_{f}$ is at most $n-1$ (i.e., $S(f)$ cannot be satisfied), completing the first part of the proof.

Now, when $f$ is not balanced then $D_{a} f$ cannot be equal to 1 for some $a$. So " $f$ not balanced and $D_{a} f \neq 0$ for any nonzero $a$ " is equivalent to " $f$ has no linear structure", completing the proof of (i). When $f$ is balanced, there exists some function in the spectrum of $f$ which is not balanced. Moreover to prove that $f$ has no linear structure is equivalent to prove that $f+\varphi_{e}$ has no linear structure, for some $e$. When $e$ is such that $g=f+\varphi_{e}$ is not balanced, $g$ has no linear structure if and only if $S(g)$ is satisfied, as remarked above.

Corollary 2 Let $f \in \mathcal{B}_{n}$ be a t-resilient function of degree $d$. Then \# $N Z_{f} \leq$ $2^{2(n-t-\epsilon-2)}$, where $N Z_{f}$ is defined by (13) and $\epsilon=\left\lfloor\frac{n-t-2}{d}\right\rfloor$. Moreover, for $n \geq 2^{2(n-t-\epsilon-2)}, f$ admits a linear structure.

Proof: By Parseval's equality and according to (2) $)$, we have: $2^{2 n}=\sum_{v \in \mathbb{F}_{2}^{n}} \mathcal{F}^{2}(f+$ $\left.\varphi_{v}\right)=2^{2(t+2+\epsilon)} \Lambda$, where clearly $\Lambda \geq \# N Z_{f}$. This implies $\# N Z_{f} 2^{2(t+2+\epsilon)} \leq 2^{2 n}$ or, equivalently, $\# N Z_{f} \leq 2^{2(n-t-\epsilon-2)}$. This proves the first part of the corollary.

To prove the second part we notice that $f$ is balanced but there always exists some $\alpha \neq 0$ such that $\mathcal{F}\left(f+\varphi_{\alpha}\right) \neq 0$. In accordance with Theorem 4, we need at least $n$ other nonzero elements, say $\left(e_{1}, \ldots, e_{n}\right)$, such that $\mathcal{F}\left(f+\varphi_{\alpha+e_{i}}\right) \neq 0$, for any $i$. But this is impossible when $n+1>\# N Z_{f}$, completing the proof.

The previous corollary implies that for certain fixed values of the parameters $n, d$ and $t$, it is impossible to construct a resilient function without linear structure. As an illustration, set $t=n-5$ and $d=3$ in Corollary 2 Then $\epsilon=1$ and $2^{2(n-t-\epsilon-2)}=2^{4}=16$. We can conclude as follows.

Corollary 3 For $n \geq 16$, any $(n-5)$-resilient function $f \in \mathcal{B}_{n}$ which is of degree 3 has a linear structure.

We conclude this section by giving a simple algorithm for checking that a function has no derivative equal to the constant function 1.

Proposition 2 Let $f \in \mathcal{B}_{n}$. Suppose that there are $u$ and $v$ in $\mathbb{F}_{2}^{n}$ such that $u \neq v \neq 0$ and the four coefficients $\mathcal{F}(f), \mathcal{F}\left(f+\varphi_{u}\right), \mathcal{F}\left(f+\varphi_{v}\right)$ and $\mathcal{F}\left(f+\varphi_{u+v}\right)$ are such that only one of them is zero. Then $f$ has no linear structure a such that $D_{a} f=1$.

Proof: The sets $Z_{f}$ and $N Z_{f}$ are respectively defined by (11) and (13). We proved that $D_{a} f \neq 1$ for all $a$ if and only if $Z_{f}$ does not contain any hyperplane (see Lemma 3 . Let $H$ be any hyperplane and let $\bar{H}$ its coset. The intersection of $H$ with any subspace $\langle u, v\rangle, u \neq v \neq 0$, is either of dimension 2 or of dimension 1 . 
If $H \subset Z_{f}$ for some $H$, then $\mathcal{F}(f)=0$ and $N Z_{f} \subset \bar{H}$. Thus, for any $u, v \in N Z_{f}, u+v \in Z_{f}$. We conclude that if a pair $(u, v)$ satisfies the hypothesis, it is impossible to have $H \subset Z_{f}$ for any $H$.

\section{Resilient Functions and Their Derivatives}

In this section, we focus on the values of the auto-correlation function of $f \in \mathcal{B}_{n}$ when $f$ is $t$-resilient. Actually we want to obtain some bounds for the absolute indicator $\mathcal{M}(f)$ (defined in $\S 2.1$ ) of such a function. We first give a general property, whose proof is given in the Appendix.

Lemma 4 Let $f \in \mathcal{B}_{n}, n \geq 3$. Assume that the weight of $f$ is even. Then

$$
\mathcal{F}\left(D_{a} f\right) \equiv 0(\bmod 8) \quad \text { for any } a \in \mathbb{F}_{2}^{n} \text {. }
$$

Remark 1 One might expect that an arbitrary $t$-resilient function satisfies the following congruence, $\mathcal{F}\left(D_{a} f\right) \equiv 0\left(\bmod 2^{t+3}\right)$. This congruence holds for $t=0$, but we easily found a 1 -resilient function $f$ such that $\mathcal{F}\left(D_{a} f\right) \equiv 0(\bmod 16)$ is not true for some $a$ (by computer).

Next we investigate how the divisibility of derivatives is related to the resiliency order, PC degree, and algebraic degree.

Theorem 5 Let $f \in \mathcal{B}_{n}$ be a t-resilient function of degree d satisfying $P C(p)$. Set $\epsilon=\left\lfloor\frac{n-t-2}{d}\right\rfloor$. Then for $p, t \geq 0$ and for any $a \in \mathbb{F}_{2}^{n}$ we have:

$$
\mathcal{F}\left(D_{a} f\right) \equiv 0 \quad\left(\bmod 2^{2 t+p+2 \epsilon+5-n}\right)
$$

This property is significant for $2 t+p+2 \epsilon+2>n$ only.

Proof: Let $a \in \mathbb{F}_{2}^{n}$ such that $w t(a)=p+1$. Let $U_{a}=\left\{v \in \mathbb{F}_{2}^{n} \mid v \preccurlyeq a\right\}$ and $\bar{a}=\left(1+a_{1}, \ldots, 1+a_{n}\right)$. Then, since $f$ satisfies $\mathrm{PC}(p)$, we can write (11) (setting $\beta=0$ and $V^{\perp}=U_{a}$ ) in the following form:

$$
\sum_{\alpha \preccurlyeq \bar{a}} \mathcal{F}^{2}\left(f+\varphi_{\alpha}\right)=2^{n-(p+1)}\left(2^{n}+\mathcal{F}\left(D_{a} f\right)\right),
$$

where $\mathcal{F}^{2}\left(f+\varphi_{\alpha}\right) \equiv 0\left(\bmod 2^{2(t+2+\epsilon)}\right)$ because $f$ is $t$-resilient (see (2)). Since $\left|\mathcal{F}\left(D_{a} f\right)\right| \leq 2^{n}$, it is easily verified that $\mathcal{F}\left(D_{a} f\right)$ is congruent to 0 modulo $2^{2 t+p+2 \epsilon+5-n}$. Thus, we have proved that (15) holds for any $a$ such that $w t(a)=$ $p+1$. Now, we proceed by induction on the weight of $a$. Assuming that (15) holds for $w t(a) \leq p+s-1, s \geq 2$ we rewrite (11) for $w t(a)=p+s$ :

$$
\sum_{\alpha \preccurlyeq \bar{a}} \mathcal{F}^{2}\left(f+\varphi_{\alpha}\right)=2^{n-(p+s)}\left(2^{n}+\sum_{u \preccurlyeq a, w t(u) \geq p+1} \mathcal{F}\left(D_{u} f\right)\right) .
$$


For convenience, let $\rho=2 t+p+2 \epsilon+5-n$. The sum on the left is congruent to 0 modulo $2^{2(t+2+\epsilon)}$. In the sum on the right, all $\mathcal{F}\left(D_{u} f\right)$ are known to be congruent to 0 modulo $2^{\rho}$ (by induction hypothesis) unless $u=a$. Hence the formula (16) has the following form: $2^{2(t+2+\epsilon)} \lambda=2^{n-(p+s)}\left(2^{\rho} \lambda^{\prime}+\mathcal{F}\left(D_{a} f\right)\right)$, for some integers $\lambda$ and $\lambda^{\prime}$. This leads to: $\mathcal{F}\left(D_{a} f\right)=2^{2(t+2+\epsilon)-n+p+s} \lambda-2^{\rho} \lambda^{\prime}=2^{\rho+s-1} \lambda-2^{\rho} \lambda^{\prime}$, since $\rho=2(t+2+\epsilon)-n+p+1$. Then we deduce that $\mathcal{F}\left(D_{a} f\right)$ is congruent to 0 modulo $2^{\rho}$ and conclude that this property holds for any $a$. Thus $\mathcal{F}\left(D_{a} f\right)$ is of the form $\pm 2^{\rho} \lambda$, for some integer $\lambda \geq 0$. Due to Lemma 4 this property is significant for $2 t+p+2 \epsilon+5-n>3$, completing the proof.

Remark 2 The first consequence of Theorem 5 is that for high order of resiliency the autocorrelation properties becomes rather poor. We proved actually that the indicators related with the propagation criterion satisfy here: $\mathcal{M}(f) \geq$ $2^{\rho}$ and $\mathcal{V}(f) \geq 2^{2 m}+2^{2 \rho} \times \mu$, where $\rho=2 t+p+2 \epsilon+5-n$ and $\mu$ denotes the number of $a \neq 0$ such that $\mathcal{F}\left(D_{a} f\right) \neq 0$.

Note that for fixed $p$ and $t$ the divisibility of derivatives depends entirely on algebraic degree $d$ via $\epsilon=\left\lfloor\frac{n-t-2}{d}\right\rfloor$. Hence the overall good cryptographic properties are exhibited only by functions of high algebraic degree. Furthermore, the congruence relation above clearly indicates that the size of derivatives is more sensitive to the changes of resiliency order $t$, than to the changes of $p$.

Now, we want to illustrate that due to the previous result a large class of resilient functions cannot be used in the design of Boolean functions having good propagation properties.

Example 2 With notation of Theorem 5, take $n=11$ and $t=3$. Then $d \leq 7$ and $\epsilon=\left\lfloor\frac{6}{d}\right\rfloor$. For any $a \in \mathbb{F}_{2}^{11}, \mathcal{F}\left(D_{a} f\right) \equiv 0\left(\bmod 2^{\rho}\right)$, where $\rho=2 t+p+2 \epsilon+5-n$. If $d=3$ then $\epsilon=2$. Applying Theorem [5, we obtain respectively for $p=$ $0,1, \ldots, 6$ the values $\rho=4,5, \ldots, 10$.

If $3<d \leq 6$ then $\epsilon=1$. We obtain respectively for $p=0,1, \ldots, 6$ the values $\rho=2,3, \ldots, 8$. Note that by Lemma 4 the results for $p=0,1$, are not significant.

Corollary 4 Let $f \in \mathcal{B}_{n}$ be a t-resilient function of degree 3 . Assume that $t=$ $n-4$. Then the derivatives of $f$ satisfy: $\mathcal{F}\left(D_{a} f\right) \equiv 0\left(\bmod 2^{n-3}\right)$ for any $a \in$ $\mathbb{F}_{2}^{n}$.

Moreover, if $f$ satisfies $P C(1)$, then $\mathcal{F}\left(D_{a} f\right) \equiv 0\left(\bmod 2^{n-2}\right)$ for any a.

Proof: Due to the Siegenthaler's upper bound, $d \leq 3$ for $t=n-4$. By setting $t=n-4, p=0$ and $d=3$ in Theorem [5, we have $\epsilon=0$ and then $2 t+p+2 \epsilon+$ $5-n=n-3$.

If $f$ satisfies $\mathrm{PC}(1)$ then $2 t+p+2 \epsilon+5-n=n-2$.

Note that for $p+t=n-2$, the result of Theorem 5 is significant for any $t \geq 0$. Taking $a$ such that $w t(a)=p+1$, we have $w t(\bar{a})=t+1$. So (1) gives here:

$$
\sum_{\alpha \preccurlyeq \bar{a}} \mathcal{F}^{2}\left(f+\varphi_{\alpha}\right)=\mathcal{F}^{2}\left(f+\varphi_{\bar{a}}\right)=2^{n-(p+1)}\left(2^{n}+\mathcal{F}\left(D_{a} f\right)\right) .
$$


Hence $\mathcal{F}^{2}\left(f+\varphi_{\bar{a}}\right)=0$ if and only if $\mathcal{F}\left(D_{a} f\right)=-2^{n}$, i.e., $a$ is a linear structure of $f$ with $D_{a} f=1$.

Corollary 5 Let $f \in \mathcal{B}_{n}$ satisfying the hypothesis of Theorem 5 with $p+t=$ $n-2$. Then

- for any $a: \mathcal{F}\left(D_{a} f\right) \equiv 0\left(\bmod 2^{t+3+2\left\lfloor\frac{p}{d}\right\rfloor}\right)$;

- for any a such that $w t(a)=p+1: \mathcal{F}^{2}(f+\varphi \bar{a})=0$ if and only if $D_{a} f=1$.

The proof of the next corollary is given in the Appendix.

Corollary 6 Let $f \in \mathcal{B}_{n}, f\left(x_{1}, \ldots, x_{n}\right)=\left(1+x_{n}\right) f_{1}\left(x_{1}, \ldots, x_{n-1}\right)+$ $x_{n} f_{2}\left(x_{1}, \ldots, x_{n-1}\right)$, where $f_{1}, f_{2}$ in $\mathcal{B}_{n-1}$. Assume that $f$ is t-resilient and satisfies $P C(p)$ with $p=n-t-2$.

Then for any $\beta \in \mathbb{F}_{2}^{n-1}$ such that $w t(\beta)=t$ and $\mathcal{F}\left(f_{1}+\varphi_{\beta}\right)=\mathcal{F}\left(f_{2}+\varphi_{\beta}\right)$, $(\beta, 1)$ is a linear structure of $f$. Furthermore, if both $f_{1}$ and $f_{2}$ are t-resilient, then $f$ is affine or constant.

\section{The Main Classes of Resilient Functions}

\subsection{Linear Concatenation}

The class of $t$-resilient functions, described by the next theorem, is actually a subclass of the Maiorana-McFarland class. It provides one of a few designs that guarantees a moderate value of nonlinearity for a given order of resiliency. We first need to introduce some notation. Let us denote by $L_{k}$ the set of all linear functions on $\mathbb{F}_{2}^{k}$; note that $\# L_{k}=2^{k}$. We define for any $0 \leq t<k$ :

$$
L_{k}^{t}=\left\{\varphi_{c}(x)=c \cdot x \mid c \in \mathbb{F}_{2}^{k}, w t(c)>t\right\} .
$$

The cardinality of $L_{k}^{t}$ is equal to $\sum_{i=0}^{k-(t+1)}\left(\begin{array}{c}k \\ t+1+i\end{array}\right)$. For fixed integers $t$ and $n$, $0 \leq t<n$, we define

$$
\mathbf{k}=\min _{t<k}\left\{k \mid \sum_{i=0}^{k-(t+1)}\left(\begin{array}{c}
k \\
t+1+i
\end{array}\right) \geq 2^{n-k}\right\} .
$$

Theorem 6 [7] For any $0 \leq t<n$, let $\mathbf{k}$ be defined by (18) and $L_{\mathbf{k}}^{t}$ by (17). Let us choose $2^{n-\mathbf{k}}$ linear functions in $L_{\mathbf{k}}^{t}$, each being labelled by an element of $\mathbb{F}_{2}^{n-\mathbf{k}}$ as follows:

$$
\tau \in \mathbb{F}_{2}^{n-\mathbf{k}} \longleftrightarrow \ell_{[\tau]} \in L_{\mathbf{k}}^{t}, \text { where }[\tau]=\sum_{i=0}^{n-\mathbf{k}-1} \tau_{i} 2^{i} .
$$

Then the Boolean function defined for all $(y, x) \in \mathbb{F}_{2}^{n-\mathbf{k}} \times \mathbb{F}_{2}^{\mathbf{k}}$ by

$$
f(y, x)=\sum_{\tau \in \mathbb{F}_{2}^{n-\mathbf{k}}}\left(y_{1}+\tau_{1}+1\right) \cdots\left(y_{n-\mathbf{k}}+\tau_{n-\mathbf{k}}+1\right) \ell_{[\tau]}(x),
$$


is a $t$-resilient function with nonlinearity $\mathcal{N}_{f}=2^{n-1}-2^{\mathbf{k}-1}$. In general deg $(f) \leq$ $n-\mathbf{k}+1$ with equality if there exists a variable $x_{i}, i=1, \ldots, \mathbf{k}$, which occurs an odd number of times in $\ell_{[\tau]}(x)$ when $\tau$ runs through $\mathbb{F}_{2}^{n-\mathbf{k}}$.

The proof of this theorem is due to Chee et al. 7]. Note that the linear functions $\ell_{[\tau]}$ in (19) are two-by-two distinct, and that, obviously, $\mathbf{k}>n / 2$. Any resilient function defined above has a simple algebraic structure, since it can be viewed as a concatenation of the linear functions $\ell_{[\tau]}$ : for any fixed value of $y$, we get $f(y, x)=\ell_{[\tau]}(x)$, where $\tau=y$. Moreover it is easy to characterize the zeros of its Walsh-spectrum and its propagation characteristics (see the next Lemma whose proof is given in the Appendix). On the one hand, these properties can be considered as a weakness. However it allows us to define precisely the cryptographic properties. We will show that a well-chosen set of functions $\ell_{[\tau]}$ insures that such a function has no linear structure.

Lemma 5 Let $f$ be a function in $\mathcal{B}_{n}$ constructed by means of Theorem [6; let $(\alpha, \beta)$ be any element in $\mathbb{F}_{2}^{n-\mathbf{k}} \times \mathbb{F}_{2}^{\mathbf{k}}$. Then $f$ satisfies:

(i) $\mathcal{F}\left(f+\varphi_{(\alpha, \beta)}\right)= \pm 2^{\mathbf{k}}$ if and only if $\varphi_{(\alpha, \beta)}=\sum_{i=1}^{n-\mathbf{k}} \alpha_{i} y_{i}+\ell_{[\tau]}(x)$ for some $\tau$. Otherwise $f+\varphi_{(\alpha, \beta)}$ is balanced.

(ii) $D_{(\alpha, \beta)} f$ is balanced if and only if $\alpha \neq 0$ or $\alpha=0$ and $D_{\beta} \ell_{[\tau]}=0$ for $2^{n-\mathbf{k}-1}$ values of $\tau$ exactly. Moreover $\mathcal{F}\left(D_{(0, \beta)}\right) \equiv 0\left(\bmod 2^{\mathbf{k}}\right)$.

(iii) $(\alpha, \beta)$ is a linear structure of $f$ if and only if $\alpha=0$ and $\ell_{[\tau]}(\beta)=c$ for all $\tau$, where $c \in \mathbb{F}_{2}$.

Remark 3 The functions defined by means of Theorem 6 are said to be threevalued, since their Fourier-spectrum has three values only, i.e., 0 and $\pm 2^{\mathbf{k}}$. They are also called three-valued almost optimal when $\mathbf{k}=(n+1) / 2$ for odd $n$ or $\mathbf{k}=(n+2) / 2$ for even $n$; in this case, the nonlinearity is maximal (for threevalued functions). Concerning the propagation characteristics, the value of the sum-of-squares indicator is known: $\mathcal{V}(f)=2^{n} \mathcal{L}^{2}(f)=2^{n+2 \mathbf{k}}$. The value of the absolute indicator depends on the choice of the functions $\ell_{[\tau]}$. More about this kind of functions can be found in [2,3].

So, it turns out that the choice of the set $\left\{\ell_{0}, \ldots, \ell_{2^{n-\mathbf{k}}}\right\}$ is crucial for propagation characteristics, especially if we want to construct resilient functions without linear structure. For clarity, we begin by giving a small example. By the next proposition, we indicate how this set can be chosen.

Example 3 Let $n=5$ and $t=0$. Thus $\sum_{i=0}^{k-1}\left(\begin{array}{c}k \\ 1+i\end{array}\right)=2^{k}-1$ and we have to choose the smallest $k$ such that $2^{k}-1 \geq 2^{5-k}$. Clearly $\mathbf{k}=3$ and we have to select $S=\left\{\ell_{0}, \ldots, \ell_{4}\right\}$, four linear functions from the set $L_{3}^{0}$. Note that $\# L_{3}^{0}=7$.

We first choose $S=\left\{x_{1}, x_{2}, x_{3}, x_{1}+x_{2}+x_{3}\right\}$. Then the function

$$
f(y, x)=\sum_{\tau \in \mathbb{F}_{2}^{2}}\left(y_{1}+\tau_{1}+1\right)\left(y_{2}+\tau_{2}+1\right) \ell_{[\tau]}(x)
$$


is a balanced function with $\mathcal{N}_{f}=12$ - according to Theorem 6 . But $(0,0,1,1,1)$ is a linear structure of $f$, since $\ell_{i}(1,1,1)=1$ for all $i, i=0, \ldots, 3$. Now we take $S=\left\{x_{1}+x_{2}, x_{1}+x_{3}, x_{2}+x_{3}, x_{1}+x_{2}+x_{3}\right\}$. It is easy to check that in this case we cannot have: $\ell_{i}(\beta)=c$ for all $i, 0 \leq i \leq 3$, and for any $\beta \in \mathbb{F}_{2}^{3}$ (where $c$ is a binary constant). Thus, $f$ has no linear structure. Furthermore, since the linear functions $\ell_{i}$ are of weight greater than one, $f$ is 1-resilient with $\mathcal{N}_{f}=12$.

Notice that the set $L_{k}^{t}$ defined by (17) has always rank $k$. Indeed, since $k>t+1$, at least the all-one vector and the $k$ vectors of weight $(k-1)$ are in $L_{k}^{t}$. By adding the all-one vector to each vector of weight $(k-1)$ we obtain the standard basis.

Proposition 3 Let $f$ be a function in $\mathcal{B}_{n}$ constructed by means of Theorem [6; so $\mathbf{k}$ and $t$ are fixed (and $\mathbf{k}>t+1$ ). Let us denote by $S$ the set of the $2^{n-\mathbf{k}}$ linear functions $\ell_{[\tau]}$ which have to be chosen in $L_{\mathbf{k}}^{t}$. Then, there is at least one choice $S$ such that $f$ has no linear structure if and only if $\mathbf{k}<2^{n-\mathbf{k}}$. In this case, $S$ can be chosen as follows:

$-\ell_{0}(x)=\lambda \cdot x$, where $\lambda$ is the all-one vector;

- for every $i, 1 \leq i \leq \mathbf{k}, \ell_{i}(x)=\left(\lambda+e_{i}\right) \cdot x$ where $e_{i}=(0, \ldots, 0, \stackrel{i}{1}, 0, \ldots, 0)$, i.e., $\left(e_{1}, \ldots, e_{\mathbf{k}}\right)$ is the standard basis;

- $\left(\ell_{\mathbf{k}+1}, \ldots, \ell_{2^{n-\mathbf{k}}-1}\right)$ are some other elements of $L_{\mathbf{k}}^{t}$.

Proof: It is a direct application of Theorem 4. (ii). Indeed $f$ is balanced and here we know exactly the nonzero coefficients of the Fourier-spectrum of $f$. So $f$ has no linear structure if and only if we can construct one $S$ such that the corresponding function $f$ satisfies the hypothesis of this theorem. From Lemma 5 . we know that $f+\varphi_{(\alpha, \beta)}$ is not balanced if and only if the function $\beta \cdot x$ is in $S$. Now we proceed as it is indicated in the proposition, and we have:

$-\ell_{0}=\lambda \cdot x$ with $w t(\lambda)=\mathbf{k}$;

- so for any basis of $\mathbb{F}_{2}^{n-\mathbf{k}}$, say $\left(\alpha_{1}, \ldots, \alpha_{n-\mathbf{k}}\right)$, the functions $f+\varphi_{\left(\alpha_{j}, \lambda\right)}$ are not balanced ;

- set $\ell_{i}(x)=\lambda \cdot x+e_{i} \cdot x, 1 \leq i \leq \mathbf{k}$ (note that $w t\left(\lambda+e_{i}\right)=\mathbf{k}-1$ ); complete the set $S$ with any other functions in $L_{\mathbf{k}}^{t}$.

Now $f$ is fully defined and we can check that, according to Theorem 4 , it has no linear structure. Set $g=f+\varphi_{(0, \lambda)}$; so $g$ is not balanced. Our construction is such that there is a basis of $\mathbb{F}_{2}^{n-\mathbf{k}} \times \mathbb{F}_{2}^{\mathbf{k}},\left(\alpha_{1}, 0\right), \ldots,\left(\alpha_{n-\mathbf{k}}, 0\right),\left(0, e_{1}\right), \ldots,\left(0, e_{\mathbf{k}}\right)$, such that the functions $g+\varphi_{\left(\alpha_{j}, 0\right)}$ and $g+\varphi_{\left(0, e_{i}\right)}$, which are respectively the functions $f+\varphi_{\left(\alpha_{j}, \lambda\right)}$ and $f+\varphi_{\left(0, \lambda+e_{i}\right)}$, are not balanced. Applying Theorem 4 , $f$ has no linear structure.

Since the rank of $L_{\mathbf{k}}^{t}$ is always equal to $\mathbf{k}$, such a construction is possible if and only if the cardinality of $S$ is strictly greater than $\mathbf{k}$, i.e., $\mathbf{k}<2^{n-\mathbf{k}}$.

Example 4 Let $n=9$. For $t=4$, we obtain $\mathbf{k}=7$ (see (18)). But, in this case, $2^{n-\mathbf{k}}=4$, implying that $f$ has always a linear structure. 
Now for $t=3$, we obtain $\mathbf{k}=6$ with $2^{n-\mathbf{k}}=8$. So we can choose $S=$ $\left\{\ell_{0}, \ldots, \ell_{8}\right\}$ in $L_{6}^{3}$, the set of linear functions $x \mapsto \beta \cdot x$ such that $\beta \in \mathbb{F}_{2}^{6}$ and $w t(\beta) \geq 4$, in such a manner that $f$ has no linear structure. According to the previous proposition, $\ell_{0}(x)=x_{1}+x_{2}+x_{3}+x_{4}+x_{5}+x_{6}$, and $\ell_{i}(x)=\ell_{0}(x)+$ $x_{i}, \quad 1 \leq i \leq 6$. We can choose $\ell_{7}$ to be any other function from $L_{6}^{3}$.

Corollary $\mathbf{7}$ For any odd $\mathbf{k} \geq 3$ it is possible to construct a $\lfloor\mathbf{k} / 2\rfloor$-resilient Boolean function $f$ of $n=2 \mathbf{k}-1$ variables of degree $\mathbf{k}$ without linear structure and with nonlinearity $\mathcal{N}_{f}=2^{n-1}-2^{\frac{n-1}{2}}$.

Proof: Since $\mathbf{k}$ is odd it is well-known that $\sum_{i=\lfloor\mathbf{k} / 2\rfloor+1}^{\mathbf{k}}\left(\begin{array}{c}\mathbf{k} \\ i\end{array}\right)=2^{\mathbf{k}-1}$. Thus, by choosing $t=\lfloor\mathbf{k} / 2\rfloor$ the cardinality of $L_{\mathbf{k}}^{t}$ is $2^{\mathbf{k}-1}$. Thus, we can construct a $t$-resilient function $f$ in $n=2 \mathbf{k}-1$ variables, with $\mathcal{N}_{f}=2^{n-1}-2^{\frac{n-1}{2}}$. Also, $\operatorname{deg}(f)=n-\mathbf{k}+1=\mathbf{k}$ since each variable $x_{i}, i=1, \ldots, \mathbf{k}$ occurs an odd number of times in $L_{\mathbf{k}}^{t}$. By Proposition $3 f$ is without linear structure.

Example 5 Let us construct $f$ by means of Corollary 7 for $\mathbf{k}=5$. So we take $t=\lfloor\mathbf{k} / 2\rfloor=2$. Since $\sum_{i=0}^{2}\left(\begin{array}{c}5 \\ 3+i\end{array}\right)=2^{4}$, \#L $L_{5}^{2}=2^{4}$, and we must take all sixteen linear functions from $L_{5}^{2}$ to construct $f$. By Corollary $7 f$ is a 2resilient function with nonlinearity $\mathcal{N}_{f}=2^{n-1}-2^{\mathbf{k}-1}=240$, and without linear structure. Furthermore, the degree of $f$ is 5 .

\subsection{Linear Structures in Recursive Constructions of Optimal Resilient Functions}

We are going to discuss two recursive constructions of resilient functions given respectively in [16] and [11]. The main interest of these constructions is that they provide optimal functions, in the sense that they have the best nonlinearity with respect to the upper bound [4. Both constructions are based on a concatenation of resilient functions with high nonlinearity. We will prove that the first construction leads to functions with linear structure while the second construction allows to avoid linear structures.

In this section, we assume that for any $t$-resilient function in $\mathcal{B}_{n}, t$ satisfies $t \geq \frac{n}{2}-2$. For this range of $t$ the upper bound on nonlinearity is $\mathcal{N}_{f} \leq 2^{n-1}-2^{t+1}$. This bound is achieved by the functions meeting the Siegenthaler's bound. Since we focus here on the existence of linear structure, we give the iterative formula proposed in [16] and indicate the nonlinearity without more explanations. By Proposition 5 (see the proof in the Appendix), we claim that such a construction in which each $f_{i}$ appears several times in the concatenation, provides functions with linear space.

Proposition 4 [16] Assume that $f_{0}(x), \ldots, f_{2^{k}-1}(x)$ are all $t$-resilient functions in $\mathcal{B}_{m}$. Let $(y, z)$ in $\mathbb{F}_{2}^{k} \times \mathbb{F}_{2}^{s}$, where $k \geq s$. Then the function $f$ defined by

$$
f(x, y, z)=\left(\sum_{\tau \in \mathbb{F}_{2}^{k}}\left(\prod_{i=1}^{s}\left(y_{i}+z_{i}+\tau_{i}\right)\right)\left(\prod_{i=s+1}^{k}\left(y_{i}+\tau_{i}\right)\right) f_{[\tau]}(x)\right)+\sum_{i=1}^{s} z_{i}
$$


is an $(t+s)$-resilient function on $\mathbb{F}_{2}^{m+k+s}$ (where the label $[\tau]$ is computed as in Theorem [6). Furthermore, if the nonlinearity of $f_{0}, \ldots, f_{2^{k}-1}$ is at least $\nu_{0}$ and the functions $f_{[\tau]}$ satisfy certain properties (see [16]) then $\mathcal{N}_{f} \geq 2^{s}\left(2^{n-1}\left(2^{k}-\right.\right.$ 1) $\left.+\nu_{0}\right)$.

Proposition 5 Let $f \in \mathcal{B}_{m+k+s}$ be a function constructed by means of Proposition 4. Then the subspace $\left\{(\alpha, \beta, \gamma) \in \mathbb{F}_{2}^{m} \times \mathbb{F}_{2}^{k} \times \mathbb{F}_{2}^{s} \mid \alpha=0, \beta=\right.$ $\left.\left(\gamma_{1}, \ldots, \gamma_{s}, 0, \ldots, 0\right)\right\} \quad$ is contained in the linear space of $f$. The linear space of $f$ has dimension at least $s$.

By an $\left(n, t, d, \mathcal{N}_{f}\right)$ function we mean an $n$-variable, $t$-resilient function $f$ with degree $d$ and nonlinearity $\mathcal{N}_{f}$. The construction introduced in [11] is a recursive one and starts with a suitable input function $f^{0}$ of type $\left(n, t, d, \mathcal{N}_{f}\right)$, which is said to be in desired form.

Definition 5 An $(n, t, d,-)$ function $f$ is in desired form if it is of the form $f=\left(1+x_{n}\right) f_{1}+x_{n} f_{2}$, where $f_{1}, f_{2}$ are $(n-1, t, d-1,-)$ functions.

An infinite sequence $f^{i}$ of $\left(n+3 i, t+2 i, d+i, \mathcal{N}_{f^{i}}=2^{n+3(i-1)+1}+4 \mathcal{N}_{f^{i-1}}\right)$ functions is then obtained for $i \geq 1$. Furthermore, if $t \geq \frac{n}{2}-2$ and $\mathcal{N}_{f^{0}}=$ $2^{n-1}-2^{t+1}$ then any function in this sequence will be optimal in the sense that its nonlinearity attains the upper bound on nonlinearity (see [11] for more details). We next describe one step of the algorithm.

Proposition 6 [1] Let $f=\left(1+x_{n}\right) f_{1}+x_{n} f_{2}$ be an $\left(n, t, d, \mathcal{N}_{f}\right)$ function in desired form, where $f_{1}, f_{2}$ are both $(n-1, t, d-1,-)$ functions. Let the functions $F$ and $G$ on $\mathbb{F}_{2}^{n+2}$ be defined by,

$$
\begin{aligned}
& F=x_{n+2}+x_{n+1}+f \\
& G=\left(1+x_{n+2}+x_{n+1}\right) f_{1}+\left(x_{n+2}+x_{n+1}\right) f_{2}+x_{n+2}+x_{n} .
\end{aligned}
$$

Then the function $H \in \mathcal{B}_{n+3}, H=\left(1+x_{n+3}\right) F+x_{n+3} G$ is an $(n+3, t+2, d+$ $\left.1,2^{n+1}+4 \mathcal{N}_{f}\right)$ function in desired form.

Proposition 7 Let $f=\left(1+x_{n}\right) f_{1}+x_{n} f_{2}$ be an $\left(n, t, d, \mathcal{N}_{f}\right)$ function in desired form. Assume that $f$ has no linear structure. Then, the function $H$ constructed by means of Proposition [6 has no linear structure.

Proof: Considering the restrictions of $H$ to the hyperplane defined by $x_{n+3}=0$ and to its coset, we note $H=(F, G)$. We will consider the restrictions of $D_{\beta} H$ to the same hyperplane and to its coset. When $\beta_{n+3}=0$, then we look at $D_{\beta} H$ with $\beta=(a, 0)$, where $a=\left(\beta_{1}, \ldots, \beta_{n+2}\right)$. But in this case $D_{\beta} H=\left(D_{a} F, D_{a} G\right)$. Thus $D_{\beta} H=c, c \in\{0,1\}$, if and only if $D_{a} F=D_{a} G=c$.

The derivative of $F$ is as follows, where $\beta^{\prime}=\left(\beta_{1}, \ldots, \beta_{n}\right): D_{\left(\beta^{\prime}, \beta_{n+1}, \beta_{n+2}\right)} F=$ $\beta_{n+1}+\beta_{n+2}+D_{\beta^{\prime}} f$. Since $f$ has no linear structure, the linear structures of $F$ 
are of the form $\left(0, \ldots, 0, \beta_{n+1}, \beta_{n+2}\right)$. So we have to compute the derivatives of $G$ with respect to $a=\left(0, \ldots, 0, \beta_{n+1}, \beta_{n+2}\right)$ :

$$
D_{a} G=\left(\beta_{n+2}+\beta_{n+1}\right)\left(f_{1}+f_{2}\right)+\beta_{n+2} .
$$

Since $f$ has no linear structure, then $f_{1}+f_{2}$ cannot be constant because $D_{(0, \ldots, 0,1)} f=f_{1}+f_{2}$. Therefore $D_{a} G=c$ if and only if $\beta_{n+2}=\beta_{n+1}=1$ (since $a \neq 0)$. But, in this case, $D_{a} G=1$ and $D_{a} F=0$; we conclude that $(a, 0)$ cannot be a linear structure of $H$.

When $\beta_{n+3}=1$, we use the general formula

$$
D_{(a, 1)} H=D_{(a, 0)} H+D_{(0, \ldots, 0,1)} H+D_{(a, 0)} D_{(0, \ldots, 0,1)} H .
$$

We obtain: $D_{(a, 1)} H=(F(x)+G(x+a), F(x+a)+G(x))$. But the function $x \mapsto F(x)+G(x+a)$ cannot be constant. Indeed $F$ is of degre $d$, where $d$ is the degree of $f$. By definition of $F$ and $G$ (see (21)) it is clear that the terms of degree $d$ in $F$ cannot be canceled in the expression of type $F(x)+G(x+a)$. Thus, $D_{(a, 1)} H$ is not constant completing the proof.

\section{References}

1. A. Canteaut and M. Trabbia, "Improved fast correlation attacks using parity-check equations of weight 4 and 5." In Advances in Cryptology - EUROCRYPT 2000, number 1807 in Lecture Notes in Computer Science, pp. 573-588, Springer-Verlag, 2000.

2. A. Canteaut, C. Carlet, P. Charpin, and C. Fontaine, "Propagation characteristics and correlation-immunity of highly nonlinear Boolean functions." In Advances in Cryptology - EUROCRYPT 2000, number 1807 in Lecture Notes in Computer Science, pp. 507-522, Springer-Verlag, 2000.

3. A. Canteaut, C. Carlet, P. Charpin, and C. Fontaine. "On cryptographic properties of the cosets of $R(1, m)$." IEEE Trans. Inform. Theory, 47(4):1494-1513, 2001.

4. C. Carlet, "On the coset weight divisibility and nonlinearity of resilient and correlation-immune functions." In Sequences and their Applications - SETA '01, Discrete Mathematics and Theoretical Computer Science, pp. 131-144. SpringerVerlag, 2001.

5. C. Carlet, "On cryptographic propagation criteria for Boolean functions." Information and Computation, number 151, pp. 32-56, 1999.

6. P. Charpin, and E. Pasalic, "On propagation characteristics of resilient functions." In Research-report RR-4537, INRIA, September 2002.

7. S. Chee, S. Lee, D. Lee, and S.H. Sung, "On the correlation immune functions and their nonlinearity." In Advances in Cryptology - ASIACRYPT'96, number 1163 in Lecture Notes in Computer Science, pp. 232-243, Springer-Verlag, 1996.

8. J. H. Evertse, "Linear structures in block ciphers." In Advances in Cryptology EUROCRYPT' 87, number 304 in Lecture Notes in Computer Science, pp. 249266, Springer Verlag, 1987.

9. M. Matsui, "Linear cryptanalysis method for DES cipher." In Advances in Cryptology - EUROCRYPT'93, number 765 in Lecture Notes in Computer Science, pp. 386-397, Springer-Verlag, 1993. 
10. W. Meier, and O. Staffelbach., "Nonlinearity criteria for cryptographic functions." In Advances in Cryptology - EUROCRYPT'93, number 434 in Lecture Notes in Computer Science, pp. 549-562, Springer-Verlag, 1988.

11. E. Pasalic, T. Johansson, S. Maitra, and P. Sarkar., "New constructions of resilient and correlation immune Boolean functions achieving upper bounds on nonlinearity." In Cryptology ePrint Archive, eprint.iacr.org, No. 2000/048, September, 2000.

12. B. Preneel, W.V. Leekwijck, L.V. Linden, R. Govaerts, and J. Vandewalle, "Propagation characteristics of Boolean functions." In Advances in Cryptology - EUROCRYPT'90, number 437 in Lecture Notes in Computer Science, pp. 155-165, Springer-Verlag, 1990.

13. P. Sarkar and S. Maitra, "Nonlinearity bounds and constructions of resilient Boolean functions." In Advances in Cryptology - EUROCRYPT 2000, number 1807 in Lecture Notes in Computer Science, pp. 515-532, Springer-Verlag, 2000.

14. T. Siegenthaler, "Correlation-immunity of nonlinear combining functions for cryptographic applications." IEEE Trans. Inform. Theory, IT-30(5): 776-780, 1984.

15. Y. Tarannikov, "On resilient Boolean functions with maximal possible nonlinearity." In Proceedings of Indocrypt 2000, number 1977 in Lecture Notes in Computer Science, pp. 19-30, Springer Verlag, 2000.

16. Y. V. Tarannikov, "New constructions of resilient Boolean functions with maximal nonlinearity." In Fast Software Encryption - FSE 2001, to be published in Lecture Notes in Computer Science, pp. 70-81 (in preproceedings). Springer Verlag, 2001.

17. A.F. Webster and S.E. Tavares, "On the design of S-boxes." In Advances in Cryptology - CRYPTO'85, number 219 in Lecture Notes in Computer Science, pp. 523-534, Springer-Verlag, 1985.

18. G. Xiao and J.L. Massey. "A spectral characterization of correlation-immune combining functions." IEEE Trans. Inform. Theory, IT-34(3):569-571, 1988.

19. X.-M. Zhang and Y. Zheng, "GAC - the criterion for global avalanche characterics of cryptographic functions." Journal of Universal Computer Science, vol. 1, no. 5, pp. 320-337, 1995.

20. X.-M. Zhang and Y. Zheng, "On relationship among avalanche, nonlinearity, and propagation criteria," In Advances in Cryptology - Asiacrypt 2000, number 1976 in Lecture Notes in Computer Science, pp. 470-483, Springer-Verlag, 2000.

\section{Appendix}

Proof of Lemma 4 Let $a$ be any nonzero word in $\mathbb{F}_{2}^{n}$, and $D_{a} f(x)=f(x)+$ $f(x+a)$. The Boolean functions $D_{a} f(x), f(x),(x+a)$ can be associated to codewords of length $2^{n}$ denoted respectively $D_{a} f, f, f_{a}$. Then we have,

$$
w t\left(D_{a} f\right)=w t(f)+w t\left(f_{a}\right)-2 w t\left(f f_{a}\right) .
$$

We note that $w t(f)=w t\left(f_{a}\right)$ for any $a$ since $f_{a}$ can be seen as a permutation of $f$. Thus the equation above may be rewritten to yield $w t\left(D_{a} f\right)=2 w t(f)-$ $2 w t\left(f f_{a}\right)$. Since by assumption $f$ is of even weight it remains to prove that $w t\left(f f_{a}\right)$ is even. We note that $\alpha \in \mathbb{F}_{2}^{n}$ satisfies $f(x) f(x+a)=1$ if and only if $\alpha+a$ satisfies it too. Thus we conclude that $w t\left(f f_{a}\right) \equiv 0(\bmod 2)$ and consequently $w t\left(D_{a} f\right) \equiv 0(\bmod 4)$. This completes the proof. 
Proof of Lemma 5. (i) For every fixed value of $y$, we get

$$
f(y, x)+\varphi_{(\alpha, \beta)}(y, x)=\ell_{[y]}(x)+\sum_{i=1}^{n-\mathbf{k}} \alpha_{i} y_{i}+\sum_{i=1}^{\mathbf{k}} \beta_{i} x_{i} .
$$

This function is balanced unless $\beta \cdot x=\ell_{[y]}(x)$. When it is not balanced, it is constant and this happens for this value of $y$ only. Indeed, assuming that it is constant for $y$, we know that $\ell_{\left[y^{\prime}\right]}(x) \neq \ell_{[y]}(x)$, for all $y^{\prime} \neq y$, providing $\mathcal{F}\left(f+\varphi_{(\alpha, \beta)}\right)= \pm 2^{\mathbf{k}}$.

(ii) We compute the derivative of $f$ with respect to $(\alpha, \beta)$ :

$$
\begin{aligned}
D_{(\alpha, \beta)} f= & f(y, x)+f(y+\alpha, x+\beta)=\sum_{\tau \in \mathbb{F}_{2}^{n-\mathbf{k}}}\left(y_{1}+\tau_{1}+1\right) \cdots\left(y_{n-\mathbf{k}}+\tau_{n-\mathbf{k}}+1\right) \ell_{[\tau]}(x) \\
+ & \sum_{\tau \in \mathbb{F}_{2}^{n-\mathbf{k}}}\left(y_{1}+\tau_{1}+\alpha_{1}+1\right) \cdots\left(y_{n-\mathbf{k}}+\tau_{n-\mathbf{k}}+\alpha_{n-\mathbf{k}}+1\right) \ell_{[\tau]}(x+\beta)= \\
& \sum_{\tau \in \mathbb{F}_{2}^{n-\mathbf{k}}}\left(y_{1}+\tau_{1}+1\right) \cdots\left(y_{n-\mathbf{k}}+\tau_{n-\mathbf{k}}+1\right)\left(\ell_{[\tau]}(x)+\ell_{[\tau+\alpha]}(x+\beta)\right) \cdot(22)
\end{aligned}
$$

For any nonzero $\alpha$, each sum $\ell_{[\tau]}(x)+\ell_{[\tau+\alpha]}(x+\beta)$ is a linear (affine) function for any $\beta$ in $\mathbb{F}_{2}^{\mathbf{k}}$ - since it is not constant by construction. So $D_{(\alpha, \beta)} f$ is balanced for any nonzero $\alpha$. When $\alpha=0$, (22) becomes,

$$
D_{(0, \beta)} f(y, x)=\sum_{\tau \in \mathbb{F}_{2}^{n-\mathbf{k}}}\left(y_{1}+\tau_{1}+1\right) \cdots\left(y_{n-\mathbf{k}}+\tau_{n-\mathbf{k}}+1\right) \ell_{[\tau]}(\beta),
$$

since $\ell_{[\tau]}(x+\beta)=\ell_{[\tau]}(x)+\ell_{[\tau]}(\beta)$. Thus $D_{(0, \beta)} f(y, x)$ is constant for any fixed $y$. We deduce: $\mathcal{F}\left(D_{(0, \beta)} f\right)=2^{\mathbf{k}} \times \sum_{\tau}(-1)^{\ell_{[\tau]}(\beta)}$.

We directly obtain (iii) from this last equality, since we have proved that $(\alpha, \beta)$ can be a linear structure when $\alpha=0$ only.

Proof of Corollary 6 Every Boolean function $f$ in $\mathcal{B}_{n}$ can be viewed as a concatenation of two functions from $\mathcal{B}_{n-1}$, called subfunctions of $f$ of dimension $n-1$. More precisely, $f$ can be written as,

$$
f\left(x_{1}, \ldots, x_{n}\right)=\left(1+x_{n}\right) f_{1}\left(x_{1}, \ldots, x_{n-1}\right)+x_{n} f_{2}\left(x_{1}, \ldots, x_{n-1}\right),
$$

for some $f_{1}, f_{2}$ in $\mathcal{B}_{n-1}$. Note that if $f$ is $t$-resilient then either both $f_{1}$ and $f_{2}$ are $(t-1)$-resilient or both $f_{1}$ and $f_{2}$ are $t$-resilient.

Let $\beta$ be a vector satisfying the condition above. The Walsh transform of $f$ in the point $(1, \beta) \in \mathbb{F}_{2}^{n}$ is calculated as,

$$
\mathcal{F}\left(f+\varphi_{(1, \beta)}\right)=\mathcal{F}\left(f_{1}+\varphi_{\beta}\right)-\mathcal{F}\left(f_{2}+\varphi_{\beta}\right) .
$$

Then, $\mathcal{F}\left(f+\varphi_{(1, \beta)}\right)=0$ due to the assumption. Since the weight of $(1, \beta)$ is $t+1$, by Corollary 5$]$ has a linear structure. 
Assume now that $f_{1}$ and $f_{2}$ are $t$-resilient. Then for any $\beta \in \mathbb{F}_{2}^{n-1}$ such that $w t(\beta)=t$ we have $\mathcal{F}\left(f_{1}+\varphi_{\beta}\right)=\mathcal{F}\left(f_{2}+\varphi_{\beta}\right)=0$. Applying Corollary 5, we conclude that, for all such $\beta,(1, \beta)$ belongs to the linear space of $f$. Then the subspace $V$, generated by all these elements in contained in the linear space of $f$. Since $0 \leq t \leq n-2$ the dimension of $V$ is at least $n-1$, completing the proof.

Proof of Proposition 5: The derivative of $f$ with respect to any direction $(0, \beta, \gamma)$ with $\beta=\left(\gamma_{1}, \ldots, \gamma_{s}, 0, \ldots, 0\right)$ is

$$
\begin{aligned}
D_{(0, \beta, \gamma)} f & =f(x, y, z)+f(x, y+\beta, z+\gamma) \\
& =\left(\sum_{\tau \in \mathbb{F}_{2}^{k}}\left(\prod_{i=1}^{s}\left(y_{i}+z_{i}+\tau_{i}\right)\right)\left(\prod_{i=s+1}^{k}\left(y_{i}+\tau_{i}\right)\right) f_{[\tau]}(x)\right)+\sum_{i=1}^{s} z_{i} \\
& +\left(\sum_{\tau \in \mathbb{F}_{2}^{k}}\left(\prod_{i=1}^{s}\left(y_{i}+\gamma_{i}+z_{i}+\gamma_{i}+\tau_{i}\right)\right)\left(\prod_{i=s+1}^{k}\left(y_{i}+\tau_{i}\right)\right) f_{[\tau]}(x)\right) \\
& +\sum_{i=1}^{s}\left(z_{i}+\gamma_{i}\right)=\sum_{i=1}^{s} z_{i}+\sum_{i=1}^{s}\left(z_{i}+\gamma_{i}\right)=\sum_{i=1}^{s} \gamma_{i} .
\end{aligned}
$$

Thus each such derivative is constant implying that the space of linear structures of $f$ has dimension at least $s$. 